\title{
El Concepto de la Economía Circular y Aplicaciones Prácticas en el Apoyo a la Educación Básica y Superior Demostrados en una Escuela Rural y una Universidad del Sur del Perú
}

"The Concept of Circular Economy and Practical Applications in

Support of Basic and Higher Education Demonstrated in a

Rural School and a University in the South of Peru"

Jesus Samuel Armacanqui-Tipacti

Universidad Nacional de Ingeniería - UNI: Lima Perú

E-mail: sarmacankit@uni.edu.pe ORCID: https://orcid.org/0000-0003-1865-876X

Doris Isabel Goicochea-Parks

Universidad Nacional Jorge Basadre Grohmann, Tacna Perú

E-mail: dgoicocheap@unjbg.edu.pe ORCID: https://orcid.org/0000-0002-5628-2153

Bertha Ofelia Saavedra-Silva

Universidad Nacional San Luis Gonzaga. Ica Perú

E-mail: ofelamica@hotmail.com ORCID: https://orcid.org/0000-0003-3497-4877

Stephany Saavedra

Universidad Alas Peruanas, Ica Perú

E-mail: stefhansaav@hotmail.com ORCID https://orcid.org/0000-003-5445-3422

Recepción: 10/06/2021 Aceptación: 30/06/2021 Publicación: 30/07/2021

\section{Resumen}

El desarrollo industrial con la Economía Lineal induce el consumo a corto plazo, la explotación de los recursos naturales, energéticos, minerales etc. La creciente demanda dificulta este modelo y trae consecuencias como incremento en costes de producción, riesgos medioambientales y otros por la acumulación ilimitada de los desechos industriales, entonces el modelo es insostenible, se requiere un nuevo enfoque acorde a las nuevas condiciones y necesidades sociales.

La Economía Circular usa las 3 R's, reducir, reutilizar y reciclar, como alternativa a 
la economía lineal, con un enfoque integral considerando la sostenibilidad ambiental, así como la atención de necesidades sociales, económicas del mundo actual. La economía circular facilita el reciclaje, las reparaciones, presentando mejores ecodiseños del ciclo de vida de los productos, basándose en innovación tecnológica, innovación sistémica, creatividad, innovación para un crecimiento sostenible, competitivo; organizativo y social.

El objetivo fue diseñar nuevas soluciones a problemas existentes aplicando la economía circular en comunidades educativas, básicas y superiores. El método utilizado se basó en el concepto del Ciclo de Vida de la Innovación, para determinar temas relevantes que respondan a necesidades existentes. Luego se desarrolló el prototipo, el cual fue mejorado y sirvió como modelo. El Resultado fue que con estudiantes primarios y Universitarios con ejemplos prácticos se aplicó la teoría de la Economía Circular de reducir, reusar y reciclar aceite residual, botellas y mangueras de plástico. Los estudiantes participantes fueron capaces de presentar soluciones nuevas a viejos problemas apoyando de manera efectiva el mejoramiento de sus competencias y aprendizaje.

\section{Palabras Clave}

Economía circular, sostenibilidad, diseño sostenible de producto, modelos de negocio sostenibles.

\section{Abstract}

Industrial development with the Linear Economy induces short-term consumption, the exploitation of natural resources, energy, minerals, etc. The growing demand makes this model difficult and brings consequences such as increased production costs, environmental risks and others due to the unlimited accumulation of industrial waste, so the model is unsustainable, a new approach is required according to the new conditions and social needs.

The Circular Economy uses the $3 \mathrm{R}$ 's, reduce, reuse and recycle, as an alternative to the linear economy, with a comprehensive approach considering environmental sustainability, as well as addressing the social and economic needs of today's world. The circular economy facilitates recycling, repairs, presenting better eco-designs of the life cycle of products, based on technological innovation, systemic innovation, creativity, innovation for sustainable, competitive growth; organizational and social. The objective was to design new solutions to existing problems applying the circular economy in educational, basic and higher communities. The method used was based on the concept of the Life Cycle of Innovation, to determine relevant issues that respond to existing needs. Then the prototype was developed, which was improved 
and served as a model. The result was that with primary and university students with practical examples, the theory of the Circular Economy was applied to reduce, reuse and recycle residual oil, plastic bottles and hoses. Participating students were able to present new solutions to old problems, effectively supporting the improvement of their skills and learning.

\section{Keywords}

Circular Economy, Reduce, Reuse, Recycle, Linear Economy, Basic Education, Higher Education, Sustainable Development, Environmental Aspect, Economic Aspect, Social Aspect.

\section{Resumo}

O desenvolvimento industrial com a Economia Linear induz ao consumo de curto prazo, à exploração de recursos naturais, energia, minerais, etc. A crescente demanda dificulta esse modelo e traz consequências como aumento dos custos de produção, riscos ambientais e outros devido ao acúmulo ilimitado de resíduos industriais, portanto o modelo é insustentável, uma nova abordagem é necessária de acordo com as novas condições e necessidades sociais.

A Economia Circular utiliza os 3 R's, reduzir, reutilizar e reciclar, como alternativa à economia linear, com uma abordagem abrangente considerando a sustentabilidade ambiental, além de atender às necessidades sociais e econômicas do mundo atual. A economia circular facilita a reciclagem, a reparação, a apresentação de melhores designs ecológicos do ciclo de vida dos produtos, com base na inovação tecnológica, inovação sistémica, criatividade, inovação para um crescimento sustentável e competitivo; organizacional e social.

O objetivo era desenhar novas soluções para os problemas existentes, aplicando a economia circular nas comunidades educacionais, básicas e superiores. 0 método utilizado baseou-se no conceito de Ciclo de Vida da Inovação, para determinar questões relevantes que respondam às necessidades existentes. Em seguida, foi desenvolvido o protótipo, que foi aprimorado e serviu de modelo. O resultado foi que com alunos do ensino fundamental e superior com exemplos práticos, a teoria da Economia Circular foi aplicada para reduzir, reaproveitar e reciclar óleo residual, garrafas plásticas e mangueiras. Os alunos participantes puderam apresentar novas soluções para velhos problemas, apoiando de forma eficaz a melhoria das suas competências e aprendizagem.

\section{Palavras Chave}

Economia Circular, Reduzir, Reutilizar, Reciclar, Economia Linear, Educação Básica, 
Ensino Superior, Desenvolvimento Sustentável, Aspecto Ambiental, Aspecto Econômico, Aspecto Social.

\section{Introducción}

Con la creciente demanda por los recursos naturales y energía, la oferta de estos recursos se hace cada vez más difícil de sostener. La exploración de los recursos naturales, tanto energéticos como minerales, se realiza en zonas más profundas y geográficamente difíciles, como el ártico o el mar profundo e inclusive se está penetrando en zonas ecológicamente sensibles Darrel, B. (2007) and Mudd, G. M. (2010). Por otra parte, la extracción se hace más costosa pues la concentración de los recursos en la roca que los contiene es cada vez menor Esto tiene dos consecuencias: por un lado, el incremento de los costos de producción y por otro lado el incremento de los riesgos medioambientales Klare, M. T. (2012), Wellmer F. and Becker-Platen J. D. (2016) y Cavallo A. J. (2004).

En estas condiciones el esquema lineal de la Producción Industrial que se caracteriza por el uso de recursos, su procesamiento, utilización y deshecho en el medio ambiente, ya no se hace sostenible. Este modelo asumía que los recursos minerales y energéticos son ilimitados. Por otra parte, asumía que el medio ambiente puede resistir la acumulación ilimitada de los desechos industriales. Esto no es sostenible y requiere de un nuevo enfoque que considere las condiciones actuales a fin de tener un modelo que sea sostenible.

\section{Estado del arte}

Múltiples trabajos acerca de la Economía Circular tratan de las aplicaciones en diferentes ámbitos, como con relación a actividades del hogar, la agricultura, la industria, mostrando ejemplos prácticos de soluciones en el contexto de las 3Rs. Sin 
embargo, pocos trabajos se relacionan a la aplicación en el rubro de la Educación. Esta tendencia se ha visto alterada en el contexto de la Pandemia del Covid-19 que ha promovido el uso de herramientas digitales y el aprendizaje remoto. Mientras que el conocimiento teórico puede realizarse utilizando las herramientas de videoconferencia, la parte experimental ha sido interrumpida, por el distanciamiento de seguridad y potencial peligro de contagio. En el presente trabajo se muestran ejemplos prácticos relacionados al uso del concepto de la Economía Circular en la Educación.

\subsection{Limitación de los Recursos Energéticos}

En la Figura 1 se presenta la producción mundial de yacimientos de Petróleo tanto mediante métodos convencionales como no-convencionales. De acuerdo con el experto Irlandés Colin Campbell la producción del crudo de los yacimientos convencionales alcanzará su máxima producción en el 2010 y luego caerá en el 2050 a 30 millones de barriles por día Darrel, B. (2007).

Fig. 1

Proyecciones en la Producción Mundial de Yacimientos Convencionales y No Convencionales

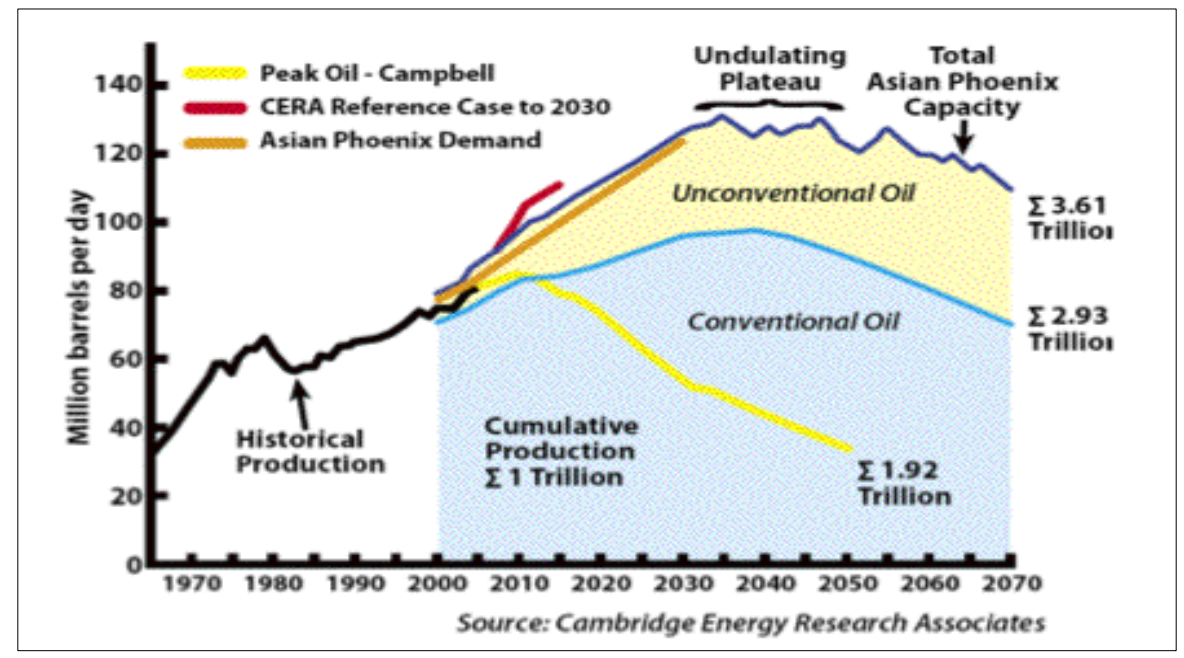

Scientific Research Journal Centro de Investigación y Desarrollo Intelectual CIDI E-ISSN: 2789-2727/ Núm. 1 Vol. 1, 37-55, Julio 2021 / www.srjournalcidi.org / https://doi.org/10.53942/srjcidi.v1i1.45 
Nota. Fuente: Darrel, B. (2007).

\subsection{Limitación de los Recursos de Minerales}

En 2017. Guiomar Calvo y otros analizan la producción histórica de 47 minerales a fin de estimar cuando alcanzaran su Producción Máxima - Production Peak. Estos investigadores utilizaron datos del USGS - US Geological Survey. EI resultado indica que para 2 de los minerales la producción máximo-teórica ya ha sido alcanzada. Para otros 12 minerales el máximo se alcanzará en los próximos 50 años mientras que la estimación para otros 30 minerales se prevé alcanzar en la próxima Centuria. Richard A. Kerr (2014) desarrolla un modelo teórico para estimar la producción global de Cobre. El resultado es que en los próximos 30 años se alcanzara el pico de producción máximo para el cobre. Los picos que se han estimado indican tanto para los recursos de petróleo como para los minerales implican que luego de alcanzar la producción máxima el pico, viene una caída de producción. Dado que se espera que el consumo continué su tendencia ascendente, habrá un déficit de oferta cada vez mayor.

Fig. 2

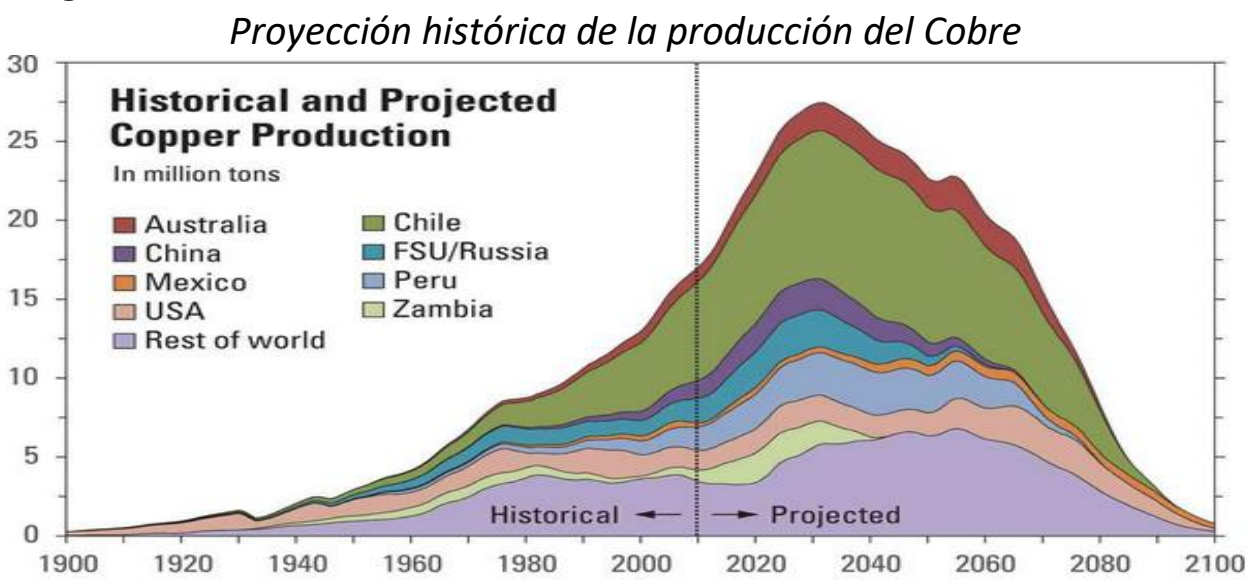

Nota. Fuente: Adaptado de S. Northey Et Al., Resources, Conservation And Recycling 83 (February 2014) (C) Published By Elsevier B.V. 


\subsection{Economía Circular y Sostenibilidad}

De acuerdo con Prieto-Sandoval, V. et. al. (2017) “la Economía Circular es un paradigma de actuación que ha evolucionado a partir del concepto de sostenibilidad y su aplicación en la economía, la sociedad, y el cuidado necesario del ambiente que nos rodea. Así, la Economía Circular se ha convertido en un paradigma que busca el desarrollo sostenible, proponiendo diferentes estrategias en toda la cadena de producción y uso de los productos y servicios". En este contexto conviene mencionar la definición de sostenibilidad. De acuerdo con un reporte el WCED World Commission on Environment and Development (1987) el desarrollo sostenible se definió como: "satisfacer las necesidades de las generaciones presentes sin comprometer las posibilidades de las generaciones del futuro para atender sus propias necesidades".

De acuerdo con la Declaración de Rio de 1992 hay tres elementos que interactúan en el desarrollo sostenible. Fig. 3 ilustra esta interrelación. Se observa que este esquema implica un enfoque a largo plazo, dado que los elementos incorporan procesos de larga duración, más allá de los procesos típicos de la producción de bienes y servicios en una fábrica o centro de trabajo.

Esto implica la necesidad de tener objetivos y planes a largo plazo, los cuales deben alinearse a planes de estado que estén por encima de las prioridades políticas partidarias que se enmarcan por lo general en el corto mediano plazo, de la duración del ejercicio del partido de turno que este en el poder ejecutivo. La consecuencia de esta escala de tiempo debe ser considerada por los organismos supranacionales para determinar objetivos y planes a los que se deban alinear los gobiernos de turno para 
evitar distraer esfuerzos que se dan por el enfoque corto a corto plazo por el que se rigen las democracias occidentales.

Fig. 3

Elementos asociados al Concepto de la Sostenibilidad

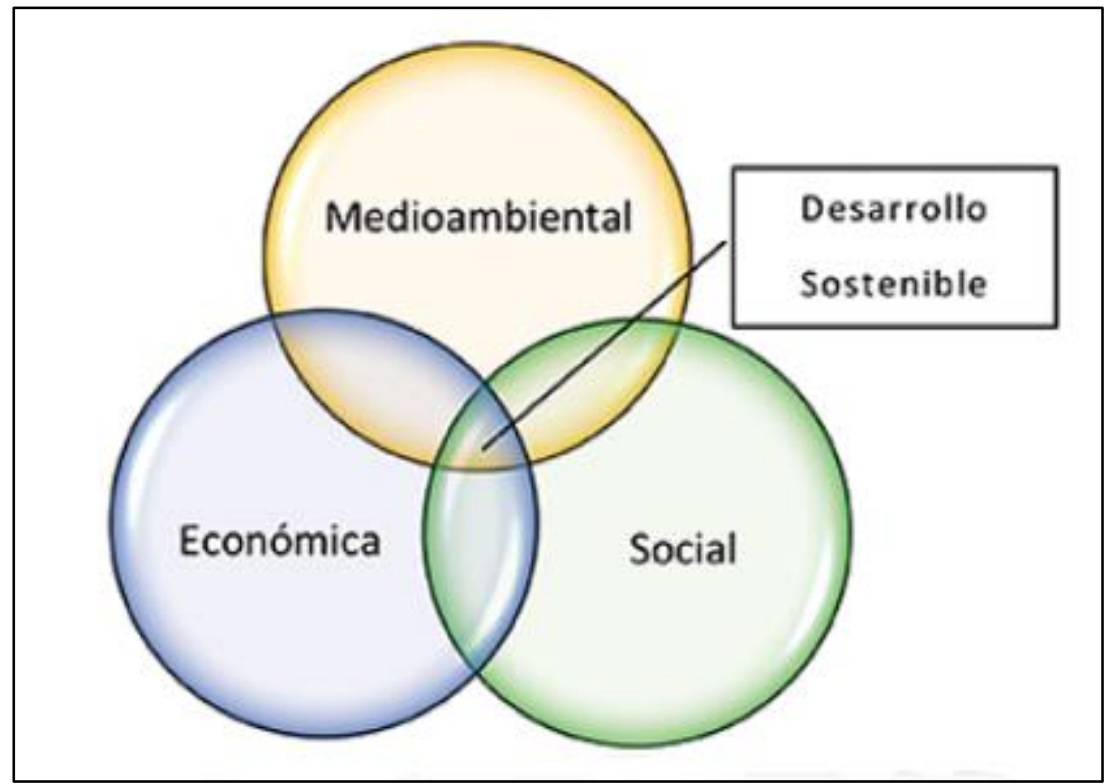

Nota. Adaptado de WCED World Commission on Environment and Development (1987).

\subsection{La Economía Circular en la Educación}

Diversos autores proponen que se establezcan acciones específicas que apoye el entendimiento del concepto de la economía circular a nivel de los centros educativos para promover aplicaciones prácticas de soluciones enmarcadas en el aspecto sostenible "Consideramos que, si pretendemos satisfacer las necesidades actuales sin comprometer la capacidad de las generaciones futuras para satisfacer sus propias necesidades, tenemos que enseñar a las generaciones actuales los principios para lograr la sostenibilidad económica, social y ambiental a corto, medio y largo plazo. Este artículo destaca el uso de actividades participativas en lugar de cursos 
tradicionales para enseñar e involucrar a estudiantes de ingeniería con las prácticas de economía circular" Ormazabal M. et. al. (2018).

\section{Metodología y Materiales}

La revisión de literatura muestra ausencia en cuanto a la implementación practica y sistemática de trabajos sobre cómo aplicar el concepto de la Economía Circular en el ámbito de la educación. Sin embargo, se aprecia que la pandemia del Coronavirus Covid-19 actúa como un catalizador para la generación de aplicaciones prácticas sobre el tema objeto del presente trabajo. La metodología utilizada en el presente trabajo toma un esquema que se ha aplicado exitosamente en la industria conocido como el Ciclo de Vida de la Innovación y las Aplicación de Nuevas Tecnologías Armacanqui J. S. (2015). En el mismo se inicia con la identificación de un problema o Necesidad Real - el "Business Need". Este primer paso requiere un conocimiento del proceso en el que se quiere plantear la innovación. El ignorar este paso podría conllevar a aplicaciones que no resulten realmente relevantes, más allá del ámbito de los que determinan el tema. Por el contrario, asegurarse que la aplicación especifica resulte en una necesidad del negocio garantiza que sea utilizado y replicado en un amplio ámbito de potenciales usuarios. A partir de allí se configuran los siguientes pasos que conllevan al diseño del prototipo y luego la aplicación a escala de campo. Es de notar que el prototipo tiene la función de probar el concepto y realizar los ajustes o mejoras necesarias. No se debe tomar el prototipo como una versión de aplicación directa, pues esa no es su función. En este prototipo se evalúan los diferentes escenarios de operación posibles con el propósito de obtener una aplicación robusta, tal como se indica en Armacanqui J. S. (2015).

Fig. 4 
El Concepto del Ciclo de Vida de La Innovación y la Nueva Tecnología.

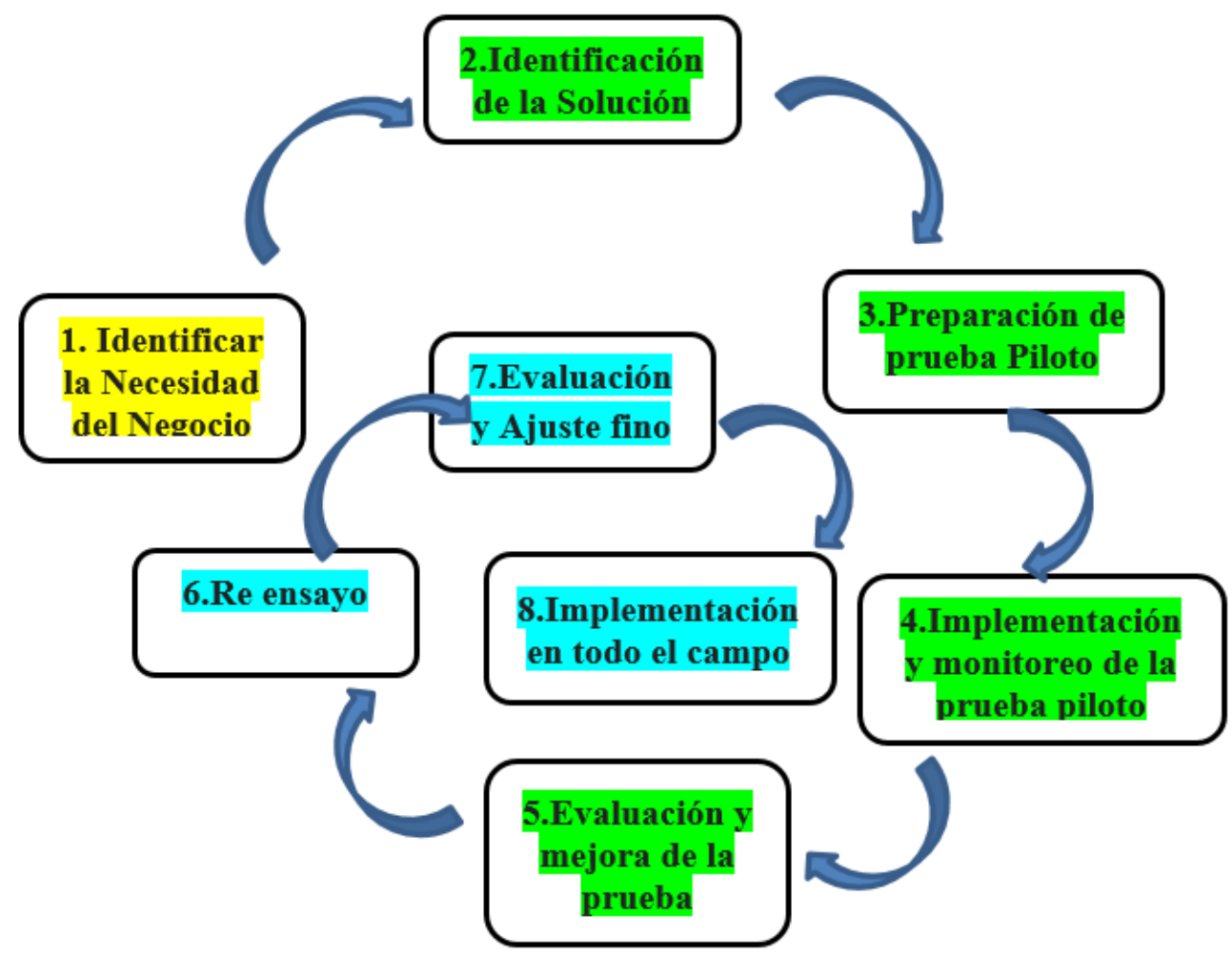

Nota. Fuente: De acuerdo con Armacanqui J. S. (2015).

De acuerdo con el esquema del Ciclo de Vida de la Innovación y la Nueva tecnología se procedió a elegir temas que representaran una "Necesidad del Negocio", es decir que sean necesidades reales del lugar ámbito de aplicación. El ciclo arriba mencionado distingue el desarrollo del Prototipo y del Modelo para la aplicación en el campo. En el primer caso se trata de tener un modelo de prueba que permita evaluar los principales factores para tener en cuenta. En el segundo caso se trata de obtener el modelo para la aplicación a mayor escala o para la masificación. Esto permitió tener un desarrollo costo efectivo y en corto tiempo. 


\section{Resultados}

A continuación, se presentan resultados de los dos ejemplos realizados. El primero para una aplicación en una escuela primaria en el Distrito de los Aquijes de Ica. EI segundo ejemplo se refiere a una aplicación realizada en el marco de una clase en la Escuela de Pregrado de la Universidad Nacional San Luis Gonzaga e Ica, en la Facultad de Ciencias Ambientales. En el primer caso el desarrollo se llevó acabo en clases presenciales. En el segundo caso el desarrollo se realizó de manera virtual y la evidencia de la ejecución se realizó mediante grabaciones de video.

\subsection{EJEMPLO No 1 - Aplicación del Concepto de la Economía Circular en una escuela rural del sur del Perú}

La educación es un proceso que muestra la necesidad de un trabajo innovador y amigable de acuerdo con el contexto que estamos viviendo, en este sentido trabajar dentro de una economía circular en un campo virtual es factible en sus diferentes niveles, siendo una herramienta digital la grabación de vídeos para el trabajo experimental, que promocione la sostenibilidad medioambiental Scarpellini, S. et. al. (2019) los temas que implementan este enfoque son los siguientes:

En el ámbito de estudiantes de Educación Primaria de una escuela multigrado de una zona rural, se logró reciclar el aceite residual de uso culinario en la elaboración de jabón sólido mediante un proceso de enfriamiento y curación, este escenario permitió aprendizajes en estudiantes dentro de una economía circular asumiendo el reciclaje. Se formó estudiantes en un contexto de educar para la vida, con un rol de líder en temas de investigación e innovación elaborando un recurso útil en este contexto de COVID19, Saavedra, B. y Armacanqui J. S. (2020).

Los materiales utilizados en la muestra inicial incluyeron $180 \mathrm{~g}$ de soda caustica, 1 litro de aceite, 1 litro de agua, envases de limpio tipo o vasos usados 
(descartables), 2 esencia de perfume y colorante (de repostería) posteriormente la segregación se incrementó a 5 litros de aceite, Saavedra. B, at. al. (2020).

La Fig. 5 muestra el esquema conceptual del ejemplo de la fabricación de jabón a partir de aceite de cocina utilizado, el cual normalmente se desecha vertiéndose al sistema de desagüe de la ciudad lo cual recarga negativamente el sistema de canalización y la laguna de oxidación.

El método utilizado se describe en las siguientes líneas.

El jabón es producido a través del proceso de frío y el curado de jabón para lo cual se utiliza como reactivo primario el hidróxido de sodio $(\mathrm{NaOH})$ cuyo nombre comercial es soda cáustica. Este reactivo es diluido en agua en una proporción de 1 litro para $180 \mathrm{gr}$ de $\mathrm{NaOH}$ hasta conseguir el proceso de enfriamiento, luego de añadir un litro de aceite previamente tamizado se pasa a un proceso de agitación (batido) por espacio de una 1 hora. Se efectúa un proceso de curado que comienza vertiendo la mezcla en los moldes que han sido reciclado o reusados, luego de 4 días una vez desmoldado se culmina el proceso de curado envolviendo el jabón en papel toalla para que permanezca durante un mes a un mes y medio, al fin de completar la reacción de saponificación, luego de lo cual el jabón se endurece y adquiere un pH menos básico. La Fig.5 muestra conceptualmente la producción obtenida a partir de 5 litros de aceite doméstico residual.

El método utilizado se basó en la implementación de soluciones prácticas a partir de materiales reciclados mediante la elaboración manual - artesanal, tal como se describe en cada uno de los ejemplos mostrados en la sección de resultados.

Fig. 5

Flujograma Conceptual del Experimento relacionado a la elaboración de jabón 
a partir de residuos de aceite de cocina.

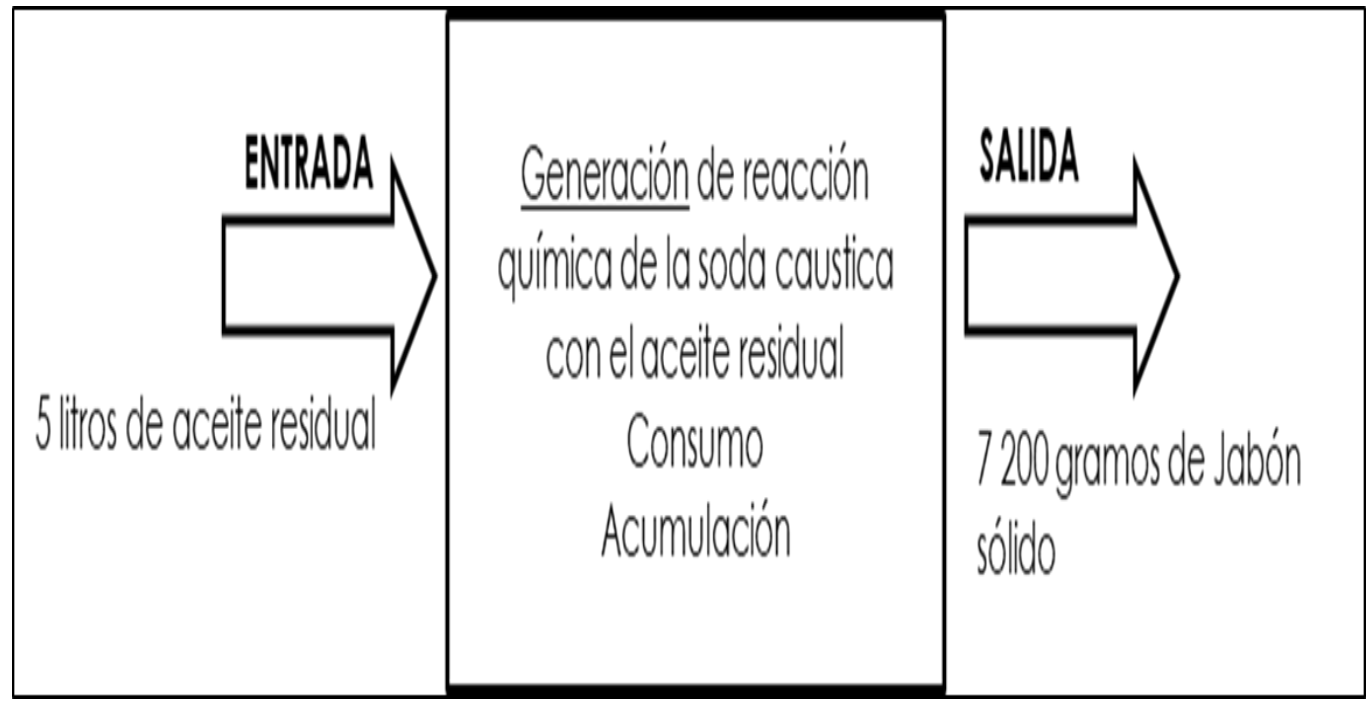

Nota. Fuente: elaboración propia.

Es de resaltar que los jabones fabricados de esta manera consideraron la parte de seguridad e higiene o cual fue necesario para el manejo seguro del componente caustico, para lo cual se utilizó un periodo de curación de varias semanas. Por otra parte, los jabones producidos fueron repartidos entre los padres y madres de familia de la comunidad del Caserío Huamanguilla del Distrito de Los Aquijes, en la Provincia de Ica en el sur del Perú, durante el inicio de la pandemia, ayudando a apoyar la higiene de los hogares involucrados.

\section{Fig. 6}

Reparto del jabón sólido a la comunidad durante el contexto de pandemia, realizado al inicio de la pandemia 


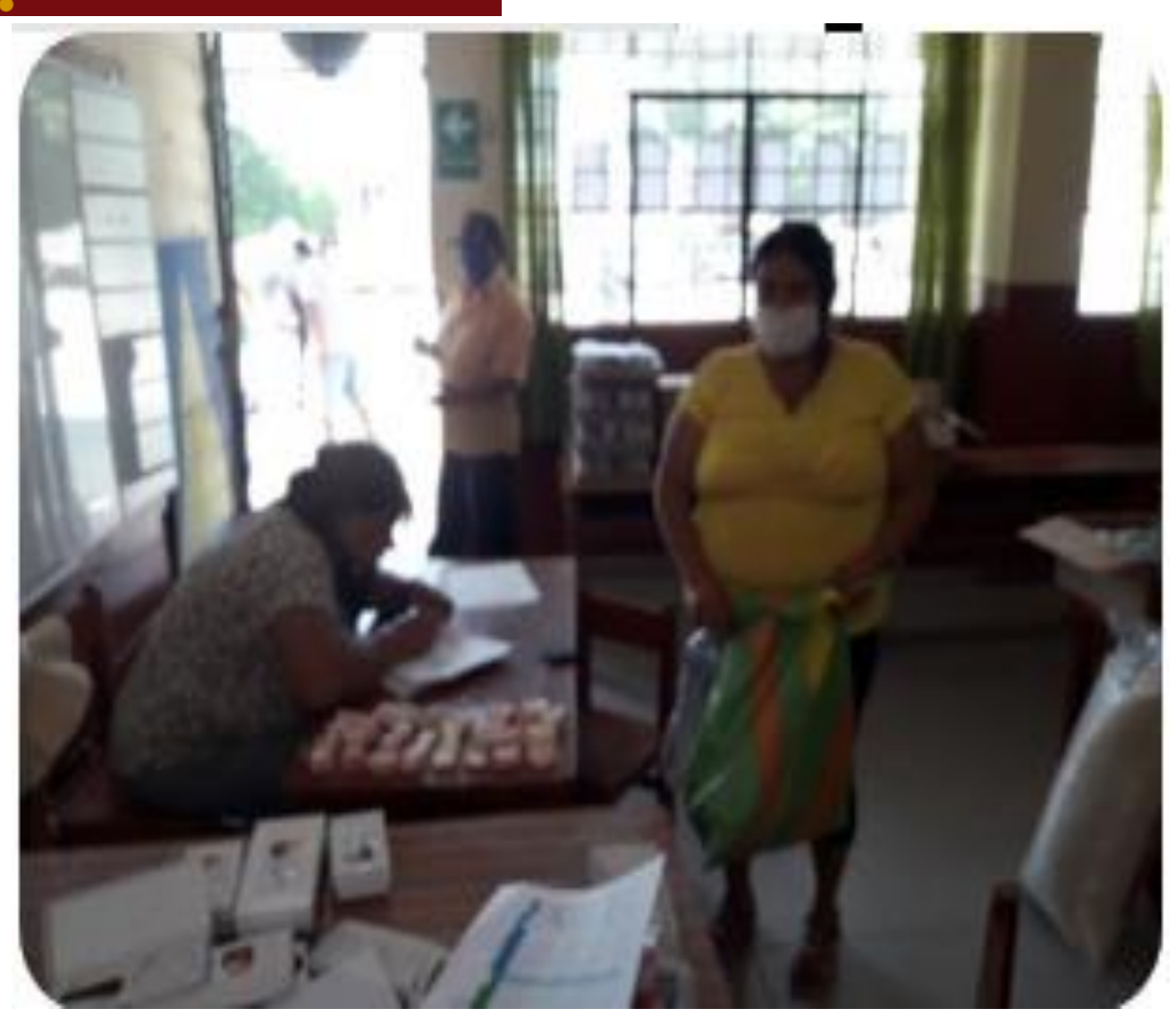

Nota: Elaboración propia.

\subsection{EJEMPLO No 2 - Aplicación del Concepto de la Economía Circular en}

\section{Centro de Educación Superior del sur del Perú.}

En el ámbito de la Educación Superior la economía circular es abordado desde la implementación de un laboratorio en los hogares de les estudiantes de la Facultad de Ingeniería Ambiental, a fin de implementar las horas de practica en la asignatura de bioquímica, a través de prácticas grabadas y subidas mediante vídeos con un instrumento de apoyo que es la hoja de trabajo.

Las practicas utilizan medios no convencionales, Saavedra. B, at. al. (2020) utilizando materiales que recirculen en lugar de ser desechados, como por ejemplo 
un foco para reemplazar el tubo de ensayo, el escalpelo por una cuchilla, un crisol por un pirex, un gotero por un frasquito vació cuenta gotas, una gradilla por una base plástica de desecho. Es de notar que entre los utensilios que hay disponibles en el hogar, por ejemplo, entre los utensilios de cocina se encuentran algunos de materiales como acero inoxidable, resistentes al calor y a los químicos agresivos, factores que también son comunes en los laboratorios de química. El mismo uso del fuego como energía calorífica para activar reacciones relacionadas a la cocción o fritura de los alimentos, se utiliza para las reacciones bioquímicas. En la Fig. 7 se ilustra un ejemplo de la Practica que utiliza una botella de un jugo de frutas comercial para reemplazar un matraz. El tema se relaciona a Los Aminoácidos y las Proteínas y su producción mediante procesos bioquímicos. Normalmente los experimentos relacionados a esta temática se realizan de manera presencial en los laboratorios de la Facultad dedicados a estas asignaturas. Sin embargo, dada las condiciones ocasionadas por la Pandemia del Covid-19 las instalaciones respectivas permanecían cerradas para observar las restricciones de seguridad.

Respecto al método utilizado, se observa en la Fig. 7 el uso de material que es fácil de manipular, cortar, pegar a condiciones de ambiente de presión y temperatura. Estos se basan en botellas y mangueras de plástico que están disponibles comercialmente o pueden ser de origen reciclados, las cuales se cortan y pegan conforme lo requiera la reacción a simular.

\section{Fig. 7}

Ilustración fotográfica de la utilización de una botella de un jugo de frutas comercial para reemplazar un matraz. 


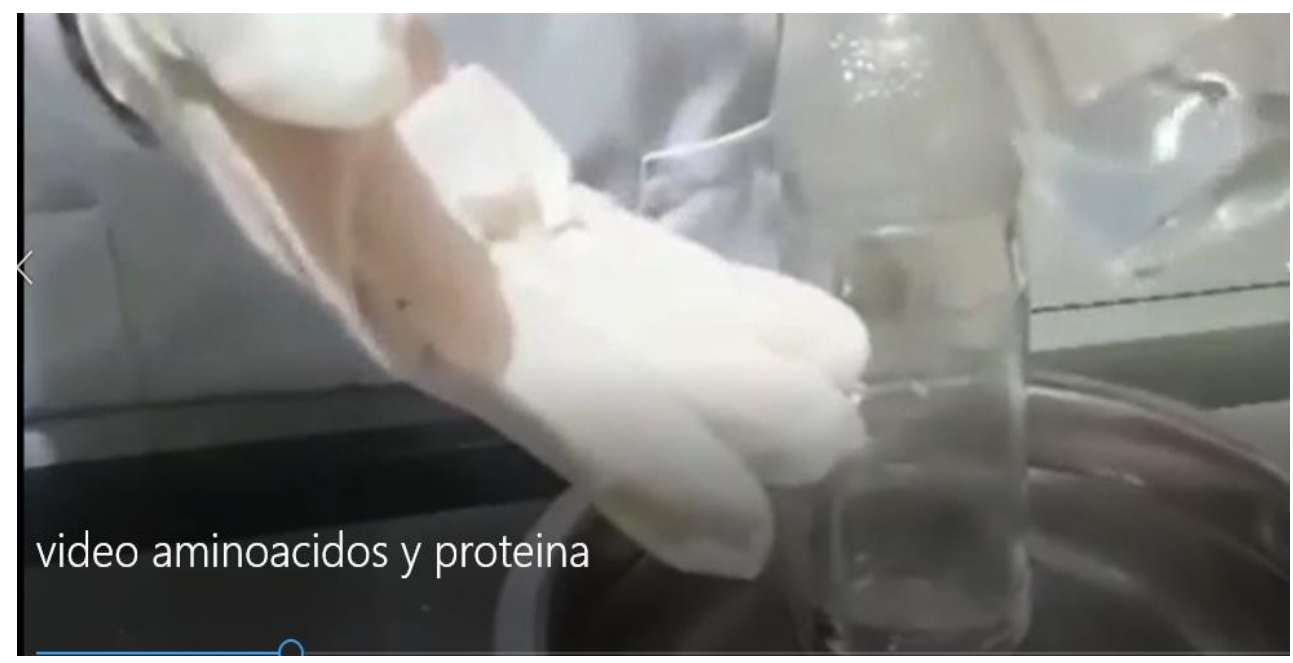

Nota: Elaboración propia.

\section{Discusión}

En el presente trabajo se presentan dos ejemplos específicos de aplicaciones prácticas en el apoyo a la Educación Básica y Superior realizados en una Escuela Rural y una Universidad del Sur del Perú, en el departamento de Ica. Ambos ejemplos son sencillos de reproducir por lo que podrían ser replicados en otros centros de enseñanza primaria o superior. Los ejemplos mencionados se alinean con el concepto de la Economía Circular de la aplicación de las 3 Rs Circular en el contexto de Reducir, Reusar y Reciclar.

\section{Conclusiones}

Se presentan dos casos específicos acerca de la aplicación del Concepto de la Economía Circular en la Educación. El primer ejemplo se relaciona a la Educación Primaria, mientras que el segundo a la Educación Superior, Con esto se demuestra que desde el ámbito educativo se puede influenciar positivamente el 
entendimiento teórico y práctico de la Economía Circular, integrándolo a la labor educativa. Estos ejemplos cumplen con los requerimientos de la Economía Circular en el contexto de Reducir, Reusar y Reciclar. Los estudiantes involucrados lograron experimentar de manera directa alternativas viables para contribuir a la Sostenibilidad de la sociedad.

Adicionalmente se observó que los alumnos participantes se sintieron empoderados a presentar soluciones originales que cumplían con los requerimientos derivados del objetivo de la aplicación de la Economía Circular con fines educativos. Igualmente es de remarcar que esta investigación realizada consolida los fundamentos teóricos impartidos durante las clases y como tal contribuye a reforzar el aprendizaje de los alumnos, utilizando métodos y materiales accesibles, seguros y en el contexto de la Economía Circular.

\section{Agradecimientos}

Se agradece a la Escuela de Educación Básica de Huamanguilla en el distrito de los Aquijes y a la Facultad de Ingeniería Ambiental de la Universidad Nacional San Luis Gonzaga de Ica.

Igualmente, especiales agradecimientos a la Sra. Flor Isabel Parks de Goicochea, por inculcar principios, valores y fomentar la creatividad que se aplican tanto en la Docencia como en los quehaceres cotidianos.

\section{Referencias}

Darrel, B. (2007). Why confusion exists over when the oil peak will occur. FEASTA (the foundation for the economics of sustainability). November del 2018. http://www.feasta.org/2007/01/12/why-confusion-exists-over-when-the-oilpeak-will-occur/ 
Mudd, G. M. (2010). The Environmental Sustainability of Mining in Australia: Key Mega-Trends and Looming Constraints. Resources Policy Volume 35, Issue 2, June 2010, Pages 98-115. Doi: 10.1016/j.resourpol.2009.12.001.

Klare, M. T. (2012). The Race for What's Left. Metropolitan Books. ISBN 9781250023971.

Wellmer F. and Becker-Platen J. D. (2016). Global Nonfuel Mineral Resources and Sustainability. U.S. Geological Survey Circular 1294 Keynote Address. https://pubs.usgs.gov/circ/2007/1294/paper1.html

Cavallo A. J. (2004). Hubbert's Petroleum Production Model: An Evaluation and Implications for World Oil Production Forecasts. Natural Resources Research, Vol. 13, No. 4, December 2004. DOI: 10.1007/s11053-004-0129-2

Calvo G. et al. (2017). Assessing maximum production peak and resource availability of non-fuel mineral resources: Analysing the influence of extractable global resources. Resources, Conservation and Recycling, Volume 125, October 2017, Pages 208-217.

https://www.sciencedirect.com/science/article/pii/S0921344917301635.

Prieto-Sandoval, V. et. al. (2017). Economía circular: Relación con la evolución del concepto de sostenibilidad y estrategias para su implementación. Memoria de Investigaciones en Ingeniería. 15, 2017, 85 - 95.

https://hdl.handle.net/10171/53653

WCED (1987). Report of the World Commission on Environment and

Development: Our Common Future Acronyms and Note on Terminology Chairman's Foreword. Oxford. New York: Oxford University Press, 1987, Brundtland, 1987.

Ormazabal M. et. al. (2018). Aumentar la participación de estudiantes de ingeniería 
en prácticas de economía circular. Memoria Investigaciones en Ingeniería. Núm. 16 (2018). http://revistas.um.edu.uy/index.php/ingenieria/article/view/308 Armacanqui J. S. (2015). The Innovations Friendly Organization: Effective Introduction of New Technologies and Innovations in Oil and Gas Companies. Paper SPE 175876. Presented at the North Africa Technical Conference and Exhibition in Cairo, Egypt 14-16 September 2015.

https://onepetro.org/SPENATC/proceedings-abstract/15NATC/115NATC/D011S006R002/183598

Saavedra B. , Armacanqui J. S. (2020). Learning Tools adapted to ecological problems through experimental simulation exercises in a Rural School in the South Pacific. ICEEL 2020: 2020 The 4th International Conference on Education and E-LearningNovember 2020 Pages 108-113

.DOI.org/10.1145/3439147.3439175

Saavedra B. O., Bendezú J. L., Tincopa J. C., Gutierrez J. O., Magallanes L. A. (2020). Water Reuse Strategy through Phyto-purification for Experiential Learning in a Rural School. ICEEL 2020: 2020 The 4th International Conference on Education and E-LearningNovember 2020 Pages 102-107.

DOI:.org/10.1145/3439147.3439174

Scarpellinin S. ,Portillo-Tarragona P. , Marco-Fondevila M. ,Marín-Vinuesa L. M., Valero-Gil J., Llera-Sastresa E. M., Zabalza-Bribián I., Llena-Macarulla, ArandaUsón (2019). Píldoras de economía circular en el aula para la enseñanza aplicada en sostenibilidad medioambiental. ZAGUAN, Repositorio institucional de documentos, Universidad de Zaragosa. DOI:10.26754/CINAIC.2019.0060 Kerr R. A. (2014). The coming copper peak. Science Magazine. 2014 Feb 14;343(6172):722-4. DOI: 10.1126/science.343.6172.722. 